\title{
Convenient two-step one-pot synthesis of 3-substituted imidazo[1,2-a]pyridines and imidazo[1,2-b]pyridazines
}

\author{
HONGLI FAN*(i) and FENGHAI LI \\ School of Chemistry and Chemical Engineering, Heze University, 2269 Daxue Road, Heze 274000, Shandong, \\ China \\ E-mail: fanhl2007@163.com
}

MS received 4 January 2018; revised 9 April 2018; accepted 10 April 2018; published online 7 May 2018

\begin{abstract}
A convenient and novel two-step one-pot method for the synthesis of 3-substituted imidazo[1,2$a$ ]pyridines and 3-substituted imidazo[1,2-b]pyridazines was developed through the reaction of heterocyclic amines and $N, N$-dimethylformamide dimethyl acetate with active electrophiles $\mathrm{RCH}_{2} \mathrm{Br}\left(\mathrm{R}=\mathrm{CO}_{2} \mathrm{Et}, \mathrm{CN}\right.$, $\mathrm{COPh}, 4$ '-MeO-PhCO and 4'-F-PhCO). This protocol provides a simple and practical approach to 3-substituted fused imidazo-heterocyclic compounds in moderate to high yields.
\end{abstract}

Keywords. Imidazo[1, 2-a]pyridine; imidazo[1, 2-b]pyridazine; heterocyclic amine; active electrophiles.

\section{Introduction}

Imidazo[1,2- $a$ ]pyridines exhibit diverse biological properties such as anticonvulsant, ${ }^{1}$ antimicrobial, ${ }^{2}$ antiviral, ${ }^{3}$ antiparasitic, ${ }^{4}$ anti-inflammatory, ${ }^{5}$ antituberculosis ${ }^{6}$ and inhibitory effects on DNA oxidation and quenching radicals. ${ }^{7}$ They are also ligands for major inhibitory neurotransmitter receptors. ${ }^{8}$ Consequently, many useful synthetic methods have been reported in the literature. Generally, such nitro-containing fused heterocycles are prepared through the condensation of heterocyclic amines with $\alpha$-haloketones in the presence of an inorganic base at high temperatures. ${ }^{9}$ This method also produces 2 -substituted products. Recently, some novel synthetic approaches have been developed to construct 3-substituted imidazo[1,2- $a$ ]pyridines. ${ }^{10}$ Although a number of investigations have been carried out, developing a convenient and library-friendly method is still desirable in hit-to-lead drug discovery, which requires quick follow-up to construct 3substituted imidazo[1,2- $a$ ]pyridines from readily accessible starting materials in a single operation under mild conditions. Herein, we report a two-step one-pot approach to the synthesis of 3-substituted imidazo[1,2a]pyridines. Commercially available 2 -aminopyridine and its derivatives were reacted with DMF-DMA $(N, N$ dimethylformamide dimethyl acetate) to produce the corresponding intermediates in near quantitative yields, which were subsequently condensed with active electrophiles, such as ethyl bromoacetate, bromoacetonitrile, 2-bromoacetophenone, 2-bromo-4'-methoxyacetophenone or 2-bromo-4'-fluoroacetophenone in the same flask without isolation to afford the desired 3substituted imidazo[1,2- $a]$ pyridines.

\section{Experimental}

\subsection{Materials and physical measurements}

All 2-aminopyridines 1a-1f, 6-chloropyridazin-3-amine 6 and active electrophiles $3 \mathbf{a}-\mathbf{3 e}$ were purchased from Nanjing Chemical Reagent Co., Ltd., (Nanjing, China, Purity more than 97\%), all reagents and solvents were used as received. Column chromatography was performed using silica gel (200-300 mesh). Nuclear Magnetic Resonance (NMR) spectra were recorded on a Varian $400 \mathrm{MHz}$ spectrometer $\left({ }^{1} \mathrm{H}: 400 \mathrm{MHz},{ }^{13} \mathrm{C}: 100 \mathrm{MHz}\right)$ at $25{ }^{\circ} \mathrm{C}$, using $\mathrm{CDCl}_{3}$ or DMSO- $d_{6}$ as the solvent. Chemical shifts $(\delta \mathrm{ppm})$ are reported with respect to tetramethylsilane (TMS) as an internal standard. Mass spectra were recorded using the Electrospray

\footnotetext{
*For correspondence

Electronic supplementary material: The online version of this article (https://doi.org/10.1007/s12039-018-1462-z) contains supplementary material, which is available to authorized users.
} 
Ionization Method (ESI) on Agilent mass spectrometers (G1969A LC/MSD TOF and 6545 Q-TOF LC/MS). Melting points were measured on a WRS-1 apparatus and are uncorrected.

\subsection{Synthesis of 3-substituted imidazo[1,2-a]pyridines $\mathbf{4 a - 4 n}$ and $\mathbf{5 a - 5 f}$}

2.2a Typical procedure for preparation of $4 \boldsymbol{a}-\mathbf{4 h}(\mathbf{4 a}$ as an example):: A solution of $0.094 \mathrm{~g} 2$-aminopyridine (1a, $1 \mathrm{mmol}$ ) and $0.24 \mathrm{~g}$ DMF-DMA ( $2 \mathrm{mmol}$ ) in $2 \mathrm{~mL}$ DMF was stirred at $65{ }^{\circ} \mathrm{C}$ for $2 \mathrm{~h}$. Then, $0.126 \mathrm{~g} \mathrm{NaHCO}_{3}(1.5$ $\mathrm{mmol})$ and $0.217 \mathrm{~g}$ ethyl bromoacetate $(\mathbf{3 a}, 1.3 \mathrm{mmol})$ were added sequentially. The mixture was stirred at $85^{\circ} \mathrm{C}$. After the reaction was completed as monitored by thin layer chromatography (TLC), it was diluted with $20 \mathrm{~mL}$ water and extracted with EtOAc $(3 \times 20 \mathrm{~mL})$. The combined organic extract was washed with water and brine, dried over anhydrous $\mathrm{Na}_{2} \mathrm{SO}_{4}$ and concentrated under reduced pressure. The crude product was purified by silica gel column chromatography (ethyl acetate/hexane, 1:6) to afford pure product $\mathbf{4 a}$.

2.2.1a Ethyl imidazo[1,2-a]pyridine-3-carboxylate $4 a:^{11}$ White solid; M.p.: $50-51{ }^{\circ} \mathrm{C} .{ }^{1} \mathrm{H}$ NMR $\left(\delta\right.$, ppm in $\mathrm{CDCl}_{3}$, $400 \mathrm{MHz}): 1.43\left(\mathrm{t}, J=7.2 \mathrm{~Hz}, 3 \mathrm{H}, \mathrm{CH}_{3}\right) ; 4.42(\mathrm{q}, J=$ $\left.7.2 \mathrm{~Hz}, 2 \mathrm{H}, \mathrm{CH}_{2}\right) ; 7.05(\mathrm{t}, J=6.8 \mathrm{~Hz}, 1 \mathrm{H}, \mathrm{ArH}) ; 7.42(\mathrm{t}, J=$ $8.4 \mathrm{~Hz}, 1 \mathrm{H}, \mathrm{ArH}) ; 7.74(\mathrm{~d}, J=8.8 \mathrm{~Hz}, 1 \mathrm{H}, \mathrm{ArH}) ; 8.31(\mathrm{~s}, 1 \mathrm{H}$, $\mathrm{ArH}) ; 9.31(\mathrm{~d}, J=6.8 \mathrm{~Hz}, 1 \mathrm{H}, \operatorname{ArH}) .{ }^{13} \mathrm{C}$ NMR $(\delta, \mathrm{ppm}$ in $\mathrm{CDCl}_{3}, 100 \mathrm{MHz}$ ): $180.58,148.35,141.42,127.59,127.39$, $117.79,115.77,114.14,60.38,14.41$. HRMS-ESI: Calcd. for $\mathrm{C}_{10} \mathrm{H}_{11} \mathrm{~N}_{2} \mathrm{O}_{2}(\mathrm{M}+\mathrm{H})^{+}:$191.0815, Found: 191.0811 .

2.2.1b Ethyl 6-bromoimidazo[1,2-a]pyridine-3-carboxylate $4 \boldsymbol{b}:{ }^{10 \mathrm{~b}}$ Light brown solid; M.p.: ${ }^{109-110}{ }^{\circ} \mathrm{C} .{ }^{1} \mathrm{H}$ NMR ( $\delta$, ppm in $\left.\mathrm{CDCl}_{3}, 400 \mathrm{MHz}\right): 1.42\left(\mathrm{t}, J=7.2 \mathrm{~Hz}, 3 \mathrm{H}, \mathrm{CH}_{3}\right)$; $4.42\left(\mathrm{q}, J=7.2 \mathrm{~Hz}, 2 \mathrm{H}, \mathrm{CH}_{2}\right) ; 7.50\left(\mathrm{dd}, J_{1}=9.2 \mathrm{~Hz}\right.$, $\left.J_{2}=1.8 \mathrm{~Hz}, 1 \mathrm{H}, \operatorname{ArH}\right) ; 7.64(\mathrm{~d}, J=9.6 \mathrm{~Hz}, 1 \mathrm{H}, \operatorname{ArH})$; $8.29(\mathrm{~s}, 1 \mathrm{H}, \mathrm{ArH}) ; 9.51(\mathrm{~d}, J=2.0 \mathrm{~Hz}, 1 \mathrm{H}, \mathrm{ArH}) .{ }^{13} \mathrm{C}$ $\operatorname{NMR}\left(\delta, \mathrm{ppm}\right.$ in $\mathrm{CDCl}_{3}, 100 \mathrm{MHz}$ ): $160.34,146.73,141.49$, $130.92,127.83,118.35,116.12,109.22,60.74,14.39$. HRMSESI: Calcd. for $\mathrm{C}_{10} \mathrm{H}_{10} \mathrm{BrN}_{2} \mathrm{O}_{2}(\mathrm{M}+\mathrm{H})^{+}:$268.9920, Found: 268.9915.

2.2.1c Ethyl 6-chloroimidazo[1,2-a]pyridine-3-carboxylate $4 c:{ }^{12}$ White solid; M.p.: $92{ }^{\circ} \mathrm{C} .{ }^{1} \mathrm{H}$ NMR $(\delta$, ppm in $\left.\mathrm{CDCl}_{3}, 400 \mathrm{MHz}\right): 1.44\left(\mathrm{t}, J=7.2 \mathrm{~Hz}, 3 \mathrm{H}, \mathrm{CH}_{3}\right) ; 4.44$ $\left(\mathrm{q}, J=7.2 \mathrm{~Hz}, 2 \mathrm{H}, \mathrm{CH}_{2}\right) ; 7.41\left(\mathrm{dd}, J_{1}=9.6 \mathrm{~Hz}, J_{2}=\right.$ $2.0 \mathrm{~Hz}, 1 \mathrm{H}, \mathrm{ArH}) ; 7.70(\mathrm{~d}, J=9.6 \mathrm{~Hz}, 1 \mathrm{H}, \mathrm{ArH}) ; 8.30(\mathrm{~s}$, $1 \mathrm{H}, \operatorname{ArH}) ; 9.41(\mathrm{~d}, J=2.4 \mathrm{~Hz}, 1 \mathrm{H}, \operatorname{ArH}) .{ }^{13} \mathrm{C} \operatorname{NMR}(\delta$, ppm in $\mathrm{CDCl}_{3}, 100 \mathrm{MHz}$ ): 160.33, 146.62, 141.69, 128.76, $125.67,122.64,118.05,116.25,60.72,14.39$. HRMS-ESI: Calcd. for $\mathrm{C}_{10} \mathrm{H}_{10} \mathrm{ClN}_{2} \mathrm{O}_{2}(\mathrm{M}+\mathrm{H})^{+}:$225.0425, Found: 225 . 0417.

2.2.1d Ethyl 6-fluoroimidazo[1,2-a]pyridine-3-carboxylate $4 d:{ }^{13}$ White solid; M.p.: $52-53{ }^{\circ} \mathrm{C} .{ }^{1} \mathrm{H}$ NMR $(\delta$, ppm in $\left.\mathrm{CDCl}_{3}, 400 \mathrm{MHz}\right): 1.42\left(\mathrm{t}, J=7.2 \mathrm{~Hz}, 3 \mathrm{H}, \mathrm{CH}_{3}\right) ; 4.42(\mathrm{q}$, $\left.J=7.2 \mathrm{~Hz}, 2 \mathrm{H}, \mathrm{CH}_{2}\right) ; 7.34\left(\mathrm{td}, J_{1}=8.8 \mathrm{~Hz}, J_{2}=2.4 \mathrm{~Hz}\right.$, $1 \mathrm{H}, \mathrm{ArH}) ; 7.71\left(\mathrm{dd}, J_{1}=9.6 \mathrm{~Hz}, J_{2}=4.8 \mathrm{~Hz}, 1 \mathrm{H}, \mathrm{ArH}\right)$; $8.30(\mathrm{~s}, 1 \mathrm{H}, \mathrm{ArH}) ; 9.28\left(\mathrm{dd}, J_{1}=4.0 \mathrm{~Hz}, J_{2}=2.4 \mathrm{~Hz}, 1 \mathrm{H}\right.$, $\mathrm{ArH}) .{ }^{13} \mathrm{CNMR}\left(\delta, \mathrm{ppm}_{\text {in }} \mathrm{CDCl}_{3}, 100 \mathrm{MHz}\right): 160.42,154.06$ $\left(\mathrm{d}, J_{C-F}=237.3 \mathrm{~Hz}\right), 145.85,141.95\left(\mathrm{~d}, J_{C-F}=2.5 \mathrm{~Hz}\right)$, $119.18\left(\mathrm{~d}, J_{C-F}=25.1 \mathrm{~Hz}\right), 118.11\left(\mathrm{~d}, J_{C-F}=8.9 \mathrm{~Hz}\right)$, $114.96\left(\mathrm{~d}, J_{C-F}=42.6 \mathrm{~Hz}\right), 109.99,60.67,14.39$. HRMSESI: Calcd. for $\mathrm{C}_{10} \mathrm{H}_{10} \mathrm{FN}_{2} \mathrm{O}_{2}(\mathrm{M}+\mathrm{H})^{+}:$209.0721, Found: 209.0713 .

2.2.1e Iimidazo[1,2-a]pyridine-3-carbonitrile $4 \boldsymbol{e}:{ }^{14} \mathrm{Wh}$ ite solid; M.p.: $160{ }^{\circ} \mathrm{C} .{ }^{1} \mathrm{H}$ NMR $\left(\delta\right.$, ppm in $\mathrm{CDCl}_{3}, 400$ MHz): $7.15(\mathrm{t}, J=7.2 \mathrm{~Hz}, 1 \mathrm{H}) ; 7.50(\mathrm{t}, J=7.2 \mathrm{~Hz}, 1 \mathrm{H})$; $7.81(\mathrm{~d}, J=9.2 \mathrm{~Hz}, 1 \mathrm{H}) ; 8.20(\mathrm{~s}, 1 \mathrm{H}) ; 8.40(\mathrm{~d} J=9.2 \mathrm{~Hz}$, 1H). ${ }^{13} \mathrm{C} \mathrm{NMR}\left(\delta\right.$, ppm in $\left.\mathrm{CDCl}_{3}, 100 \mathrm{MHz}\right): 147.20,142.47$, 128.32, 125.66, 118.69, 115.09, 111.13, 98.20. HRMS-ESI: Calcd. for $\mathrm{C}_{8} \mathrm{H}_{6} \mathrm{~N}_{3}(\mathrm{M}+\mathrm{H})^{+}:$: 144.0556, Found: 144.0550.

2.2.1f (Imidazo[1,2-a]pyridin-3-yl)phenylmethanone 4f: $10 \mathrm{~b}$ White solid; M.p.: $110-111{ }^{\circ} \mathrm{C} .{ }^{1} \mathrm{H}$ NMR $(\delta$, ppm in DMSO- $\left.d_{6}, 400 \mathrm{MHz}\right): 7.33(\mathrm{t}, J=7.4 \mathrm{~Hz}, 1 \mathrm{H}) ; 7.57(\mathrm{t}$, $J=7.6 \mathrm{~Hz}, 2 \mathrm{H}) ; 7.68(\mathrm{~m}, 2 \mathrm{H}) ; 7.87(\mathrm{t}, J=7.4 \mathrm{~Hz}, 3 \mathrm{H}) ; 8.24$ $(\mathrm{s}, 1 \mathrm{H}) ; 9.63(\mathrm{~d}, J=7.2 \mathrm{~Hz}, 1 \mathrm{H}) .{ }^{13} \mathrm{C} \mathrm{NMR}\left(\delta, \mathrm{ppm}\right.$ in $\mathrm{CDCl}_{3}$, $100 \mathrm{MHz}$ ): $184.82,149.05,145.63,139.24,132.04,129.45$, $128.88,128.83,128.59,123.52,117.73,115.15$. HRMSESI: Calcd. for $\mathrm{C}_{14} \mathrm{H}_{11} \mathrm{~N}_{2} \mathrm{O}(\mathrm{M}+\mathrm{H})^{+}$: 223.0866, Found: 223.0856.

2.2.1g (Imidazo[1,2-a]pyridin-3-yl)(4'-methoxyphenyl) methanone 4g: ${ }^{10 \mathrm{~b}}$ White solid; M.p.: $113-114{ }^{\circ} \mathrm{C} .{ }^{1} \mathrm{H}$ NMR $\left(\delta\right.$, ppm in $\left.\mathrm{CDCl}_{3}, 400 \mathrm{MHz}\right): 3.91\left(\mathrm{~s}, 3 \mathrm{H}, \mathrm{CH}_{3}\right) ; 7.03(\mathrm{~d}, J=$ $8.8 \mathrm{~Hz}, 2 \mathrm{H}, \mathrm{ArH}) ; 7.26(\mathrm{t}, J=7.2 \mathrm{~Hz}, 1 \mathrm{H}, \mathrm{ArH}) ; 7.67(\mathrm{t}, J=$ $8.4 \mathrm{~Hz}, 1 \mathrm{H}, \mathrm{ArH}) ; 7.89(\mathrm{~d} J=8.8 \mathrm{~Hz}, 2 \mathrm{H}, \mathrm{ArH}) ; 7.99(\mathrm{~d}, J=$ $9.2 \mathrm{~Hz}, 1 \mathrm{H}, \mathrm{ArH}) ; 8.25$ (s, 1H, ArH); 9.71 (d, $J=6.8 \mathrm{~Hz}$, $1 \mathrm{H}, \mathrm{ArH}) .{ }^{13} \mathrm{C} \mathrm{NMR}\left(\delta, \mathrm{ppm}\right.$ in $\left.\mathrm{CDCl}_{3}, 100 \mathrm{MHz}\right): 183.40$, $163.45,146.53,140.41,131.20,131.01,130.94,129.04$, 123.21, 116.63, 116.15, 114.14, 55.61. HRMS-ESI: Calcd. for $\mathrm{C}_{15} \mathrm{H}_{13} \mathrm{~N}_{2} \mathrm{O}_{2}(\mathrm{M}+\mathrm{H})^{+}:$: 253.0972, Found: 253.0947 .

2.2.1h (4'-Fluorophenyl)(imidazo[1,2-a]pyridin-3-yl)methanone 4h: ${ }^{10 \mathrm{~b}}$ White solid; M.p.: $132-133{ }^{\circ} \mathrm{C} .{ }^{1} \mathrm{H}$ NMR ( $\delta$, ppm in $\left.\mathrm{CDCl}_{3}, 400 \mathrm{MHz}\right): 7.16-7.26(\mathrm{~m}, 3 \mathrm{H}) ; 7.60(\mathrm{t}$, $J=7.8 \mathrm{~Hz}, 1 \mathrm{H}) ; 7.83(\mathrm{~d} J=8.8 \mathrm{~Hz}, 1 \mathrm{H}) ; 7.90-7.95(\mathrm{~m}, 2 \mathrm{H}) ;$ $8.21(\mathrm{~s}, 1 \mathrm{H}) ; 9.73(\mathrm{~d}, J=6.8 \mathrm{~Hz}, 1 \mathrm{H}) .{ }^{13} \mathrm{C} \mathrm{NMR}(\delta, \mathrm{ppm}$ in DMSO- $\left.d_{6}, 100 \mathrm{MHz}\right): 182.32 ; 164.37\left(\mathrm{~d}, J_{C-F}=198.6 \mathrm{~Hz}\right)$; $148.54 ; 145.36 ; 135.31 ; 131.56\left(\mathrm{~d}, J_{C-F}=7.2 \mathrm{~Hz}\right) ; 130.17$; $128.45 ; 122.81 ; 117.52 ; 115.76\left(\mathrm{~d}, J_{C-F}=17.4 \mathrm{~Hz}\right) ; 115.74$. HRMS-ESI: Calcd. for $\mathrm{C}_{14} \mathrm{H}_{10} \mathrm{FN}_{2} \mathrm{O}(\mathrm{M}+\mathrm{H})^{+}:$: 241.0772, Found: 241.0757.

\section{2b Typical procedure for preparation of $4 \boldsymbol{i}-\mathbf{4 n}(\mathbf{4} \boldsymbol{i}$ as} an example): A solution of $0.173 \mathrm{~g} 2$-amino-5-bromopyridi ne $(\mathbf{1 b}, 1 \mathrm{mmol})$ and $0.24 \mathrm{~g}$ DMF-DMA $(2 \mathrm{mmol})$ in $2 \mathrm{~mL}$ DMF was stirred at $65{ }^{\circ} \mathrm{C}$ for $3 \mathrm{~h}$. Then, $0.126 \mathrm{~g} \mathrm{NaHCO}_{3}$ $(1.5 \mathrm{mmol}), 0.033 \mathrm{~g} \mathrm{KI}(0.2 \mathrm{mmol})$ and $0.156 \mathrm{~g}$ bromoacetonitrile $(\mathbf{3 b}, 1.3 \mathrm{mmol})$ were added sequentially. The mixture was stirred at $85{ }^{\circ} \mathrm{C}$. After the reaction was completed as monitored by TLC, it was diluted with $20 \mathrm{~mL}$ water and extracted with $\mathrm{CHCl}_{3}(3 \times 20 \mathrm{~mL})$. The combined organic extract was washed with water, dried over anhydrous $\mathrm{Na}_{2} \mathrm{SO}_{4}$ and concentrated under reduced pressure. The crude product was purified by silica gel column chromatography (chloroform/hexane, 3:1) to afford pure product $4 \mathbf{i}$.

2.2.2a 6-Bromoimidazo[1,2-a]pyridine-3-carbonitrile 4i: 10d White solid; M.p.: $194-196{ }^{\circ} \mathrm{C} .{ }^{1} \mathrm{H}$ NMR $(\delta$, ppm in 
$\left.\mathrm{CDCl}_{3}, 400 \mathrm{MHz}\right): 7.47\left(\mathrm{dd}, J_{1}=9.8 \mathrm{~Hz}, J_{2}=2.0 \mathrm{~Hz}, 1 \mathrm{H}\right)$; $7.74(\mathrm{~d}, J=9.6 \mathrm{~Hz}, 1 \mathrm{H}) ; 8.17(\mathrm{~s}, 1 \mathrm{H}) ; 8.42(\mathrm{~d}, J=2.4 \mathrm{~Hz}$, 1H). ${ }^{13} \mathrm{C} \mathrm{NMR}\left(\delta, \mathrm{ppm}\right.$ in $\left.\mathrm{CDCl}_{3}, 100 \mathrm{MHz}\right): 145.85,142.66$, 132.00, 125.81, 119.20, 110.52, 110.08, 98.54. HRMS-ESI: Calcd. for $\mathrm{C}_{8} \mathrm{H}_{5} \mathrm{BrN}_{3}(\mathrm{M}+\mathrm{H})^{+}: 221.9661$, Found: 221.9652. 2.2.2b 6-Chloroimidazo[1,2-a]pyridine-3-carbonitrile 4j: ${ }^{15}$ Light yellow solid; M.p.: $195-198{ }^{\circ} \mathrm{C} .{ }^{1} \mathrm{H}$ NMR $(\delta, \mathrm{ppm}$ in $\left.\mathrm{CDCl}_{3}, 400 \mathrm{MHz}\right): 7.53\left(\mathrm{dd}, J_{1}=9.6 \mathrm{~Hz}, J_{2}=1.8 \mathrm{~Hz}\right.$, $1 \mathrm{H}) ; 7.68(\mathrm{~d}, J=9.6 \mathrm{~Hz}, 1 \mathrm{H}) ; 8.15(\mathrm{~s}, 1 \mathrm{H}) ; 8.52(\mathrm{~d}$, $J=1.6 \mathrm{~Hz}, 1 \mathrm{H}) .{ }^{13} \mathrm{C} \mathrm{NMR}\left(\delta, \operatorname{ppm}\right.$ in $\left.\mathrm{CDCl}_{3}, 100 \mathrm{MHz}\right)$ : $145.66,142.77,132.00,129.90,123.64,119.20,118.96$, 110.45, 109.99. HRMS-ESI: Calcd. for $\mathrm{C}_{8} \mathrm{H}_{5} \mathrm{ClN}_{3}(\mathrm{M}+\mathrm{H})^{+}$: 178.0167, Found: 178.0158.

2.2.2c 6-fluoroimidazo[1,2-a]pyridine-3-carbonitrile $\mathbf{4 k}$ : 16 White solid; M.p.: $173-176{ }^{\circ} \mathrm{C}$. ${ }^{1} \mathrm{H}$ NMR $(\delta$, ppm in DMSO- $\left.d_{6}, 400 \mathrm{MHz}\right): 7.70\left(\mathrm{td}, J_{1}=10.0 \mathrm{~Hz}, J_{2}=2.0 \mathrm{~Hz}\right.$, $1 \mathrm{H}) ; 7.92\left(\mathrm{dd}, J_{1}=9.8 \mathrm{~Hz}, J_{2}=5.2 \mathrm{~Hz}, 1 \mathrm{H}\right) ; 8.48(\mathrm{~s}$, $1 \mathrm{H}) ; 8.98(\mathrm{t}, J=3.0 \mathrm{~Hz}, 1 \mathrm{H}) .{ }^{13} \mathrm{C} \mathrm{NMR}\left(\delta, \mathrm{ppm}\right.$ in $\mathrm{CDCl}_{3}$, $100 \mathrm{MHz}): 154.50\left(\mathrm{~d}, J_{C-F}=241.6 \mathrm{~Hz}\right), 144.79,143.02$ $\left(\mathrm{d}, J_{C-F}=2.4 \mathrm{~Hz}\right), 120.43\left(\mathrm{~d}, J_{C-F}=25.1 \mathrm{~Hz}\right), 119.23$ $\left(\mathrm{d}, J_{C-F}=8.9 \mathrm{~Hz}\right), 112.99\left(\mathrm{~d}, J_{C-F}=41.2 \mathrm{~Hz}\right), 110.53$, 99.61. HRMS-ESI: Calcd. for $\mathrm{C}_{8} \mathrm{H}_{5} \mathrm{FN}_{3}(\mathrm{M}+\mathrm{H})^{+}$: 162.0462 , Found: 162.0459.

2.2.2d (6-Bromoimidazo[1,2-a]pyridin-3-yl)phenylmetha none 4l: ${ }^{10 \mathrm{~b}}$ White solid; M.p.: $143-145{ }^{\circ} \mathrm{C} .{ }^{1} \mathrm{H} \operatorname{NMR}(\delta$, ppm in $\left.\mathrm{CDCl}_{3}, 400 \mathrm{MHz}\right): 7.57(\mathrm{t}, J=7.6 \mathrm{~Hz}, 2 \mathrm{H})$; $7.67(\mathrm{t}, J=7.2 \mathrm{~Hz}, 1 \mathrm{H}) ; 7.79-7.86(\mathrm{~m}, 4 \mathrm{H}) ; 8.25(\mathrm{~s}$, $1 \mathrm{H}) ; 9.70(\mathrm{~s}, 1 \mathrm{H}) .{ }^{13} \mathrm{C} \mathrm{NMR}\left(\delta, \mathrm{ppm}\right.$ in $\left.\mathrm{CDCl}_{3}, 100 \mathrm{MHz}\right)$ : $184.70,147.34,145.40,138.78,132.70,132.29,128.96$, 128.80, 128.65, 123.53, 118.20, 110.05. HRMS-ESI: Calcd. for $\mathrm{C}_{14} \mathrm{H}_{10} \mathrm{BrN}_{2} \mathrm{O}(\mathrm{M}+\mathrm{H})^{+}: 300.9971$, Found: 300.9955.

2.2.2e (6-Chloroimidazo[1,2-a]pyridin-3-yl)phenylmetha none 4m: ${ }^{10 \mathrm{c}}$ White solid; M.p.: $138{ }^{\circ} \mathrm{C} .{ }^{1} \mathrm{H}$ NMR $(\delta, \mathrm{ppm}$ in DMSO- $\left.d_{6}, 400 \mathrm{MHz}\right): 7.59(\mathrm{t}, J=7.4 \mathrm{~Hz}, 2 \mathrm{H}) ; 7.69(\mathrm{t}$, $J=7.4 \mathrm{~Hz}, 1 \mathrm{H}) ; 7.78\left(\mathrm{dd}, J_{1}=9.2 \mathrm{~Hz}, J_{2}=2.0 \mathrm{~Hz}\right.$, $1 \mathrm{H}) ; 7.88(\mathrm{~d}, J=7.2 \mathrm{~Hz}, 2 \mathrm{H}) ; 7.94(\mathrm{~d}, J=9.6 \mathrm{~Hz}, 1 \mathrm{H})$; $8.31(\mathrm{~s}, 1 \mathrm{H}) ; 9.68(\mathrm{~d}, J=1.6 \mathrm{~Hz}, 1 \mathrm{H}) .{ }^{13} \mathrm{C} \operatorname{NMR}(\delta, \mathrm{ppm}$ in DMSO- $\left.d_{6}, 100 \mathrm{MHz}\right): 183.78,146.75,145.38,138.26$, 132.36, 130.49, 128.78, 128.72, 126.01, 123.16, 122.32, 118.26. HRMS-ESI: Calcd. for $\mathrm{C}_{14} \mathrm{H}_{10} \mathrm{ClN}_{2} \mathrm{O}(\mathrm{M}+\mathrm{H})^{+}$: 257.0476, Found: 257.0454.

2.2.2f (6-Fluoroimidazo[1,2-a]pyridin-3-yl)phenylmetha none 4n: ${ }^{10 \mathrm{c}}$ Light yellow solid; M.p.: $131-132{ }^{\circ} \mathrm{C} .{ }^{1} \mathrm{H}$ NMR $\left(\delta, \mathrm{ppm}\right.$ in DMSO- $\left.d_{6}, 400 \mathrm{MHz}\right): 7.58(\mathrm{t}, J=7.6 \mathrm{~Hz}, 2 \mathrm{H})$; $7.68(\mathrm{t}, J=7.4 \mathrm{~Hz}, 1 \mathrm{H}) ; 7.80\left(\mathrm{td}, J_{1}=8.6 \mathrm{~Hz}, J_{2}=2.4 \mathrm{~Hz}\right.$, $1 \mathrm{H}) ; 7.87(\mathrm{~d}, J=8.4 \mathrm{~Hz}, 2 \mathrm{H}) ; 7.97\left(\mathrm{dd}, J_{1}=9.6 \mathrm{~Hz}\right.$, $\left.J_{2}=5.2 \mathrm{~Hz}, 1 \mathrm{H}\right) ; 8.30(\mathrm{~s}, 1 \mathrm{H}) ; 9.62\left(\mathrm{dd}, J_{1}=5.0 \mathrm{~Hz}, J_{2}=\right.$ $2.6 \mathrm{~Hz}, 1 \mathrm{H}) .{ }^{13} \mathrm{C} \mathrm{NMR}\left(\delta, \mathrm{ppm}\right.$ in $\left.\mathrm{CDCl}_{3}, 100 \mathrm{MHz}\right)$ : 184.72 ; $154.80\left(\mathrm{~d}, J_{C-F}=239.7 \mathrm{~Hz}\right) ; 145.14 ; 143.56\left(\mathrm{~d}, J_{C-F}=\right.$ $1.7 \mathrm{~Hz}) ; 138.38 ; 132.62 ; 128.80\left(\mathrm{~d}, J_{C-F}=2.9 \mathrm{~Hz}\right) ; 121.94$ $\left(\mathrm{d}, J_{C-F}=24.9 \mathrm{~Hz}\right) ; 117.48\left(\mathrm{~d}, J_{C-F}=9.7 \mathrm{~Hz}\right) ; 116.601$; 116.172. HRMS-ESI: Calcd. for $\mathrm{C}_{14} \mathrm{H}_{10} \mathrm{FN}_{2} \mathrm{O}(\mathrm{M}+\mathrm{H})^{+}$: 241.0772, Found: 241.0767.

2.2c Typical procedure for preparation of $5 a-5 f(5 a$ as an example): A solution of $0.11 \mathrm{~g}$ 2-amino-3-hydroxypyridine (1e, $1 \mathrm{mmol})$ and $0.24 \mathrm{~g}$ DMF-DMA $(2 \mathrm{mmol})$ in
$2 \mathrm{~mL}$ DMF was stirred at $65{ }^{\circ} \mathrm{C}$ for $2 \mathrm{~h}$. Then, $0.252 \mathrm{~g}$ $\mathrm{NaHCO}_{3}(3 \mathrm{mmol})$ and $0.417 \mathrm{~g}$ ethyl bromoacetate (3a, 2.5 mmol) were added sequentially. The mixture was stirred at $85^{\circ} \mathrm{C}$. After the reaction was completed as monitored by thin layer chromatography (TLC), it was diluted with $20 \mathrm{~mL}$ water and extracted with $\mathrm{CHCl}_{3}(3 \times 30 \mathrm{~mL})$. The combined organic extract was washed with water, dried over anhydrous $\mathrm{Na}_{2} \mathrm{SO}_{4}$ and concentrated under reduced pressure. The crude product was purified by silica gel column chromatography (chloroform/hexane, 1:1) to afford pure product 5 a.

2.2.3a Ethyl 8-(2-ethoxy-2-oxoethoxy)H-imidazo[1,2-a] pyridine-3-carboxylate 5a: White solid, M.p.: $93-95{ }^{\circ} \mathrm{C} .{ }^{1} \mathrm{H}$ $\operatorname{NMR}\left(\delta, p p m\right.$ in $\left.\mathrm{CDCl}_{3}, 400 \mathrm{MHz}\right): 1.29(\mathrm{t}, J=7.2 \mathrm{~Hz}, 3 \mathrm{H}$, $\left.\mathrm{CH}_{3}\right), 1.43\left(\mathrm{t}, J=7.2 \mathrm{~Hz}, 3 \mathrm{H}, \mathrm{CH}_{3}\right), 4.28(\mathrm{q}, J=7.2 \mathrm{~Hz}$, $\left.2 \mathrm{H}, \mathrm{CH}_{2}\right), 4.42$ (q, J = 7.2 Hz, 2H, $\left.\mathrm{CH}_{2}\right), 4.98\left(\mathrm{~s}, 2 \mathrm{H}, \mathrm{CH}_{2}\right)$, $6.72(\mathrm{~d}, J=7.6 \mathrm{~Hz}, 1 \mathrm{H}, \mathrm{ArH}), 6.93(\mathrm{t}, J=7.4 \mathrm{~Hz}, 1 \mathrm{H}, \mathrm{ArH})$, 8.26 (s, 1H, ArH), 9.00 (d, $J=6.8 \mathrm{~Hz}, 1 \mathrm{H}, \mathrm{ArH}) .{ }^{13} \mathrm{C} \mathrm{NMR}$ ( $\delta$, ppm in $\left.\mathrm{CDCl}_{3}, 100 \mathrm{MHz}\right): 168.11,160.57,147.02,142.34$, 140.37, 121.44, 116.87, 113.88, 107.16, 66.28, 61.51, 60.51, 14.40, 14.11. HRMS-ESI: Calcd. for $\mathrm{C}_{14} \mathrm{H}_{17} \mathrm{~N}_{2} \mathrm{O}_{5}(\mathrm{M}+\mathrm{H})^{+}$: 293.1132, Found: 293.1123.

2.2.3b 8-(Cyanomethoxy)H-imidazo[1,2-a]pyridine-3-ca rbonitrile 5b: Light brown solid; M.p.: $229-230{ }^{\circ} \mathrm{C} .{ }^{1} \mathrm{H}$ NMR $\left(\delta, \mathrm{ppm}\right.$ in DMSO- $\left.d_{6}, 400 \mathrm{MHz}\right): 5.47\left(\mathrm{~s}, 2 \mathrm{H}, \mathrm{CH}_{2}\right), 7.23(\mathrm{~m}$, $2 \mathrm{H}, \mathrm{ArH}), 8.39$ (d, J = 5.2 Hz, 1H, ArH), 8.43 (s, 1H, ArH). ${ }^{13} \mathrm{C}$ NMR $\left(\delta, \mathrm{ppm}\right.$ in DMSO- $\left.d_{6}, 100 \mathrm{MHz}\right): 145.96,142.68$, 140.85, 121.56, 116.40, 115.42, 111.67, 109.08, 99.22, 54.99. HRMS-ESI: Calcd.. for $\mathrm{C}_{10} \mathrm{H}_{7} \mathrm{~N}_{4} \mathrm{O}(\mathrm{M}+\mathrm{H})^{+}:$199.0614, Found: 199.0608 .

2.2.3c 2-(3-BenzoylH-imidazo[1,2-a]pyridin-8-yloxy)-1phenylethanone 5c: White solid, M.p.: 209-211 ${ }^{\circ} \mathrm{C} .{ }^{1} \mathrm{H}$ NMR $\left(\delta\right.$, ppm in DMSO- $\left.d_{6}, 400 \mathrm{MHz}\right): 5.97\left(\mathrm{~s}, 2 \mathrm{H}, \mathrm{CH}_{2}\right), 7.20(\mathrm{~m}$, $2 \mathrm{H}, \mathrm{ArH}), 7.60(\mathrm{t}, J=7.6 \mathrm{~Hz}, 4 \mathrm{H}, \mathrm{ArH}), 7.71(\mathrm{~m}, 2 \mathrm{H}$, ArH), $7.90(\mathrm{~d}, J=7.2 \mathrm{~Hz}, 2 \mathrm{H}, \operatorname{ArH}), 8.06(\mathrm{~d}, J=7.6 \mathrm{~Hz}$, 2H, ArH), 8.19 (s, 1H, ArH), $9.28(\mathrm{~d}, J=6.8 \mathrm{~Hz}, 1 \mathrm{H}$, ArH). ${ }^{13} \mathrm{C}$ NMR $\left(\delta\right.$, ppm in DMSO- $\left.d_{6}, 100 \mathrm{MHz}\right): 194.19$, $184.39,147.38,144.49,142.71,139.16,134.54,134.44$, $132.64,129.35,129.16,128.36,124.11,121.53,116.12$, 109.65, 71.45. HRMS-ESI: Calcd. for $\mathrm{C}_{22} \mathrm{H}_{17} \mathrm{~N}_{2} \mathrm{O}_{3}(\mathrm{M}+$ $\mathrm{H})^{+}$: 357.1234, Found: 357.1225.

2.2.3d Ethyl6-bromo-8-(2-ethoxy-2-oxoethoxy)H-imidaz o[1,2-a]pyridine-3-carboxylate 5d: White solid, M.p.: 92$94{ }^{\circ} \mathrm{C} .{ }^{1} \mathrm{H}$ NMR $\left(\delta\right.$, ppm in $\left.\mathrm{CDCl}_{3}, 400 \mathrm{MHz}\right): 1.31(\mathrm{t}$, $\left.J=7.0 \mathrm{~Hz}, 3 \mathrm{H}, \mathrm{CH}_{3}\right), 1.43\left(\mathrm{t}, J=7.0 \mathrm{~Hz}, 3 \mathrm{H}, \mathrm{CH}_{3}\right)$, $4.30\left(\mathrm{q}, J=7.2 \mathrm{~Hz}, 2 \mathrm{H}, \mathrm{CH}_{2}\right), 4.43(\mathrm{q}, J=7.2 \mathrm{~Hz}$, $\left.2 \mathrm{H}, \mathrm{CH}_{2}\right), 4.97\left(\mathrm{~s}, 2 \mathrm{H}, \mathrm{CH}_{2}\right), 6.81$ (s, 1H, ArH), 8.21 (s, $1 \mathrm{H}, \mathrm{ArH}), 9.17$ (s, $1 \mathrm{H}, \mathrm{ArH}) .{ }^{13} \mathrm{C} \mathrm{NMR}\left(\delta, \mathrm{ppm}\right.$ in $\mathrm{CDCl}_{3}$, $400 \mathrm{MHz}$ ): 167.60, 160.29, 146.66, 140.95, 140.27, 121.59, 117.03, 111.40, 108.55, 66.42, 61.80, 60.80, 14.36, 14.11. HRMS-ESI: Calcd. for $\mathrm{C}_{14} \mathrm{H}_{16} \mathrm{BrN}_{2} \mathrm{O}_{5}(\mathrm{M}+\mathrm{H})^{+}: 371.0237$, Found: 371.0231.

2.2.3e 6-Bromo-8-(cyanomethoxy)H-imidazo[1,2-a]pyrid ine-3-carbonitrile 5e : Light brown solid, M.p.: $207-208{ }^{\circ} \mathrm{C}$. ${ }^{1} \mathrm{H}$ NMR $(\delta$, ppm in DMSO-d 6 , $400 \mathrm{MHz}): 5.50\left(\mathrm{~s}, 2 \mathrm{H}, \mathrm{CH}_{2}\right)$, 7.46 (s, 1H, ArH), 8.43 (s, 1H, ArH), 8.66 (s, 1H, ArH). ${ }^{13} \mathrm{C}$ NMR $\left(\delta, \mathrm{ppm}\right.$ in DMSO- $\left.d_{6}, 100 \mathrm{MHz}\right): 145.70,142.83$, 


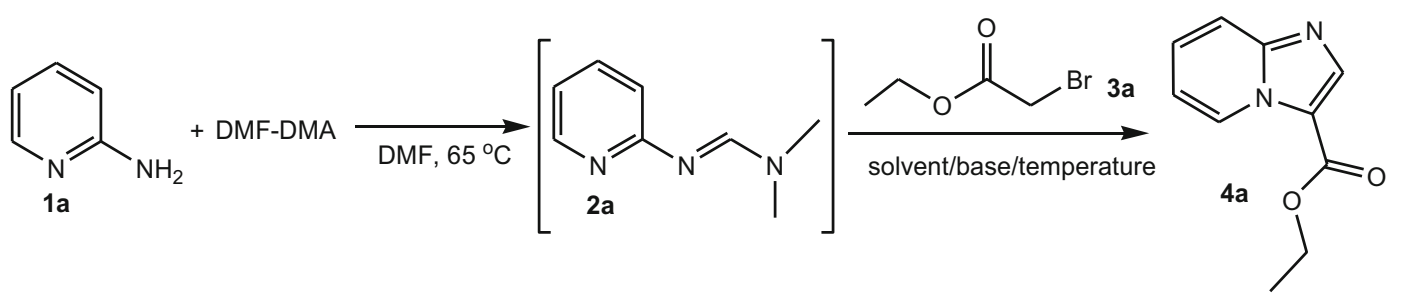

Scheme 1. Synthesis of ethyl imidazo[1,2- $a]$ pyridine-3-carboxylate 4 a.

139.63, 121.70, 116.11, 112.31, 111.16, 108.89, 99.79, 55.29. HRMS-ESI: Calcd. for $\mathrm{C}_{10} \mathrm{H}_{6} \mathrm{BrN}_{4} \mathrm{O}(\mathrm{M}+\mathrm{H})^{+}:$: 276.9719, Found: 276.9715.

2.2.3f 2-(3-Benzoyl-6-bromoH-imidazo[1,2-a]pyridin-8-y loxy)-1-phenylethanone $5 f$ : Light yellow solid, M.p.: 212$215^{\circ} \mathrm{C}$. ${ }^{1} \mathrm{H}$ NMR $\left(\delta\right.$, ppm in DMSO- $\left.d_{6}, 400 \mathrm{MHz}\right): 6.01(\mathrm{~s}$, $\left.2 \mathrm{H}, \mathrm{CH}_{2}\right), 7.51$ (s, 1H, ArH), 7.61 (t, $\left.J=7.6 \mathrm{~Hz}, 4 \mathrm{H}, \mathrm{ArH}\right)$, $7.71(\mathrm{~m}, 2 \mathrm{H}, \mathrm{ArH}), 7.90$ (d, $J=7.2 \mathrm{~Hz}, 2 \mathrm{H}, \mathrm{ArH}), 8.06$ (d, $J=7.2 \mathrm{~Hz}, 2 \mathrm{H}, \mathrm{ArH}), 8.21$ (s, 1H, ArH), 9.42(s, 1H, ArH). ${ }^{13} \mathrm{C}$ NMR $\left(\delta, \mathrm{ppm}\right.$ in DMSO- $\left.d_{6}, 100 \mathrm{MHz}\right): 193.50,184.50$, $147.52,144.25,141.47,138.81,134.55,134.40,132.86$, $129.26,129.19$, 128.43, 124.25, 121.17, 112.64, 109.83, 71.84. HRMS-ESI: Calcd. for $\mathrm{C}_{22} \mathrm{H}_{16} \mathrm{BrN}_{2} \mathrm{O}_{3}(\mathrm{M}+\mathrm{H})^{+}$: 435.0339, Found: 435.0332.

2.2d Typical procedure for preparation of 3-substituted imidazo[1,2-b]pyridazines $\mathbf{8 a - 8 c ( 8 a}$ as an example): A solution of $0.130 \mathrm{~g}$ 6-chloropyridazin-3-amine (6, $1 \mathrm{mmol}$ ) and $0.24 \mathrm{~g}$ DMF-DMA (2 mmol) in $2 \mathrm{~mL} \mathrm{DMF}$ was stirred at $65{ }^{\circ} \mathrm{C}$ for $3 \mathrm{~h}$. Then, $0.126 \mathrm{~g} \mathrm{NaHCO}_{3}(1.5 \mathrm{mmol})$, $0.033 \mathrm{~g} \mathrm{KI}(0.2 \mathrm{mmol})$ and $0.217 \mathrm{~g}$ ethyl bromoacetate (3a, $1.3 \mathrm{mmol}$ ) were added sequentially. The mixture was stirred at $85^{\circ} \mathrm{C}$. After the reaction was completed as monitored by TLC, it was diluted with $20 \mathrm{~mL}$ water and extracted with $\mathrm{CHCl}_{3}$ $(3 \times 20 \mathrm{~mL})$. The combined organic extract was washed with water, dried over anhydrous $\mathrm{Na}_{2} \mathrm{SO}_{4}$ and concentrated under reduced pressure. The crude product was purified by silica gel column chromatography (chloroform/hexane, 3:1) to afford pure product $\mathbf{8 a}$.

2.2.4a Ethyl 6-chloroimidazo[1,2-b]pyridazine-3-carboxylate 8a: Light brown solid; M.p: $92-93{ }^{\circ} \mathrm{C} .{ }^{1} \mathrm{H}$ NMR $(\delta, \mathrm{ppm}$ in $\left.\mathrm{CDCl}_{3}, 400 \mathrm{MHz}\right): 1.45$ (t, $\left.J=7.2 \mathrm{~Hz}, 3 \mathrm{H}, \mathrm{CH}_{3}\right) ; 4.48$ (q, $\left.J=7.0 \mathrm{~Hz}, 2 \mathrm{H}, \mathrm{CH}_{2}\right) ; 7.28(\mathrm{~d}, J=9.2 \mathrm{~Hz}, 1 \mathrm{H}, \mathrm{ArH}) ; 8.03$ $(\mathrm{d}, J=9.6 \mathrm{~Hz}, 1 \mathrm{H}, \mathrm{ArH}) ; 8.39$ (s, $1 \mathrm{H}, \mathrm{ArH}) .{ }^{13} \mathrm{C} \mathrm{NMR}(\delta$, ppm in $\mathrm{CDCl}_{3}, 100 \mathrm{MHz}$ ): 158.20, 148.38, 140.93, 140.67, 127.40, 121.51, 120.48, 61.08, 14.33. HRMS-ESI: Calcd. for $\mathrm{C}_{9} \mathrm{H}_{9} \mathrm{ClN}_{3} \mathrm{O}_{2}(\mathrm{M}+\mathrm{H})^{+}:$226.0378, Found: 226.0377 .

2.2.4b 6-Chloroimidazo[1,2-b]pyridazine-3-carbonitrile $\boldsymbol{8 b}:{ }^{11}$ Light yellow solid; M.p.: $143-144{ }^{\circ} \mathrm{C} .{ }^{1} \mathrm{H}$ NMR $(\delta$, ppm in $\mathrm{CDCl}_{3}, 400 \mathrm{MHz}$ ): 7.34 (d, $\left.J=9.6 \mathrm{~Hz}, 1 \mathrm{H}\right) ; 8.07$ $(\mathrm{d}, J=9.6 \mathrm{~Hz}, 1 \mathrm{H}) ; 8.26(\mathrm{~s}, 1 \mathrm{H}) .{ }^{13} \mathrm{C} \mathrm{NMR}(\delta, \mathrm{ppm}$ in $\mathrm{CDCl}_{3}, 100 \mathrm{MHz}$ ): 159.83, 149.39, 141.72, 139.61, 127.74, 123.00, 109.12. HRMS-ESI: Calcd. for $\mathrm{C}_{7} \mathrm{H}_{4} \mathrm{ClN}_{4}(\mathrm{M}+\mathrm{H})^{+}$: 179.0119, Found: 179.0113 .

2.2.4c (6-Chloroimidazo[1,2-b]pyridazin-3-yl)(phenyl)me thanone 8c: ${ }^{10 \mathrm{a}}$ Light yellow solid; M.p.: $257-258{ }^{\circ} \mathrm{C} .{ }^{1} \mathrm{H}$ $\operatorname{NMR}\left(\delta, \mathrm{ppm}\right.$ in DMSO- $\left.d_{6}, 400 \mathrm{MHz}\right): 7.59(\mathrm{t}, J=7.4 \mathrm{~Hz}$, $2 \mathrm{H}) ; 7.69(\mathrm{t}, J=9.6 \mathrm{~Hz}, 2 \mathrm{H}) ; 7.99(\mathrm{~d}, J=7.2 \mathrm{~Hz}$,
$2 \mathrm{H}) ; 8.28(\mathrm{~s}, 1 \mathrm{H}) ; 8.44(\mathrm{~d}, J=9.6 \mathrm{~Hz}, 1 \mathrm{H}) .{ }^{13} \mathrm{C} \mathrm{NMR}(\delta$, ppm in $\mathrm{CDCl}_{3}, 100 \mathrm{MHz}$ ): 182.53, 149.32, 140.31, 140.19, 137.69, 133.36, 129.43, 128.79, 126.95, 126.85, 123.59. HRMS-ESI: Calcd. for $\mathrm{C}_{13} \mathrm{H}_{9} \mathrm{ClN}_{3} \mathrm{O}(\mathrm{M}+\mathrm{H})^{+}:$258.0429, Found: 258.0420.

\section{Results and Discussion}

\subsection{Optimization of the reaction conditions of $\mathbf{4 a}$}

Initially, we found that treatment of 2-aminopyridine 1 with DMF-DMA in DMF at $65^{\circ} \mathrm{C}$ resulted in the formation of $(E)-N, N$-dimethy- $N^{\prime}$-(pyridin-2-yl)formamidine $\mathbf{2 a}$ clearly by TLC in consistence with literature report. ${ }^{17}$

Then, we turned our attention to the optimization of reaction conditions of the second step, namely the cyclization process between the intermediate $\mathbf{2 a}$ and ethyl bromoacetate $\mathbf{3 a}$, to establish an efficient two-step one-pot procedure for the synthesis of ethyl imidazo[1,2-a]pyridine-3-carboxylate 4a (Scheme 1). As shown in Table 1, different bases and solvents were investigated at varied temperatures. The reaction was found to proceed smoothly in the presence of 1.5 equivalent of $\mathrm{NaHCO}_{3}$ in $\mathrm{DMF}$ at $85^{\circ} \mathrm{C}$, delivering the product $4 \mathbf{a}$ in $83 \%$ yield (Table 1, entry 1). Decrease or increase in temperature had no positive effect on the reaction efficiency (Table 1, entry 2-3). Switching to other solvents such as EtOH and dioxane, led to inferior results (Table 1, entries 4-5). Other inorganic and organic bases were also found to promote this transformation albeit in relatively low yields (Table 1, entry 6-12). Taken together, we found that the optimal protocol for the second step of preparing $\mathbf{4 a}$ was to conduct the reaction in $\mathrm{DMF}$ at $85^{\circ} \mathrm{C}$ with 1.5 equivalent of $\mathrm{NaHCO}_{3}$ as a base. The complete synthesis of $\mathbf{4 a}$ was adopted as a two-step one-pot procedure.

\subsection{Synthesis of 3-substituted imidazo[1,2-a]pyridines $\mathbf{4 a - 4 n}$}

With the above optimized two-step one-pot procedure, the reactions of 2-aminopyridine 1 a and DMF-DMA with several active electrophiles $\mathrm{RCH}_{2} \mathrm{Br}$ 
Table 1. Optimization of the reaction conditions for the second-step condensation of $\mathbf{4} \mathbf{a}^{\mathrm{a}}$.

\begin{tabular}{|c|c|c|c|c|c|}
\hline Entry & Base & Solvent & Time $(\mathrm{h})^{\mathrm{b}}$ & Temperature $\left({ }^{\mathrm{o}} \mathrm{C}\right)^{\mathrm{c}}$ & Yield $(\%)^{\mathrm{d}}$ \\
\hline 1 & $\mathrm{NaHCO}_{3}$ & DMF & 1 & 85 & 83 \\
\hline 2 & $\mathrm{NaHCO}_{3}$ & DMF & 1.5 & 75 & 81 \\
\hline 3 & $\mathrm{NaHCO}_{3}$ & DMF & 1 & 95 & 79 \\
\hline 4 & $\mathrm{NaHCO}_{3}$ & $\mathrm{EtOH}$ & 3 & reflux & 63 \\
\hline 5 & $\mathrm{NaHCO}_{3}$ & Dioxane & 1.5 & 85 & 65 \\
\hline 6 & $\mathrm{Na}_{2} \mathrm{CO}_{3}$ & DMF & 2 & 85 & 70 \\
\hline 7 & $\mathrm{Na}_{2} \mathrm{CO}_{3}$ & $\mathrm{EtOH}$ & 3 & reflux & 51 \\
\hline 8 & $\mathrm{Na}_{2} \mathrm{CO}_{3}$ & Dioxane & 2 & 85 & 56 \\
\hline 9 & $\mathrm{~K}_{2} \mathrm{CO}_{3}$ & DMF & 1 & 85 & 65 \\
\hline 10 & $\mathrm{NaOH}$ & DMF & 1 & 85 & 47 \\
\hline 11 & $\mathrm{C}_{5} \mathrm{H}_{5} \mathrm{~N}$ & DMF & 7 & 85 & 39 \\
\hline 12 & $\mathrm{Et}_{3} \mathrm{~N}$ & DMF & 5 & 85 & 46 \\
\hline
\end{tabular}

${ }^{\text {a }}$ Reaction conditions: 2-Aminopyridine $1 \mathbf{a}(1.0 \mathrm{mmol})$ and DMF-DMA (2.0 equiv) were mixed and stirred in DMF (2 mL) at $65{ }^{\circ} \mathrm{C}$ for $2 \mathrm{~h}$. Then, ethyl bromoacetate $3 \mathrm{a}$ (1.3 equiv) and base (1.5 equiv) were added and the stirring continued for additional $1-7 \mathrm{~h}$. ${ }^{\mathrm{b}}$ Reaction time for the second step. ${ }^{\mathrm{c}}$ Reaction temperature for the second step. ${ }^{\mathrm{d}}$ Isolated yield based on $1 \mathbf{a}$.

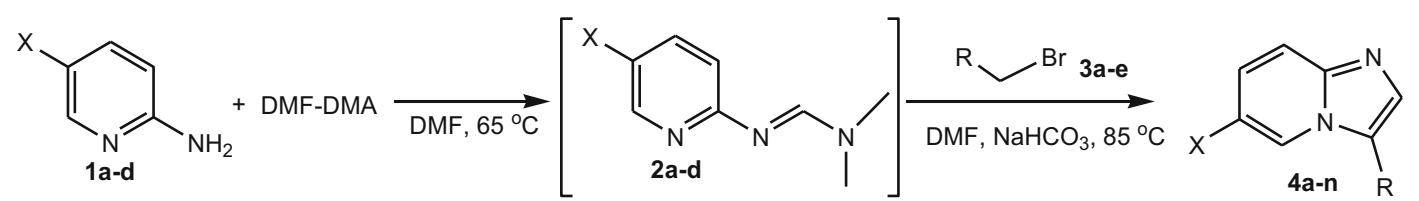

Scheme 2. Synthesis of 3-substituted imidazo[1,2-a]pyridines 4a-4n.

$\left(\mathrm{R}=\mathrm{CN}, \mathrm{CO}_{2} \mathrm{Et}, \mathrm{COPh}, 4^{\prime}-\mathrm{MeO}-\mathrm{PhCO}\right.$ and 4'-F-PhCO) 3a-3e were carried out successfully to give corresponding 3-substituted imidazo[1,2-a]pyridines $\mathbf{4 a}, \mathbf{4 e - 4 h}$ in good yields (Scheme 2, Table 2, entry 1, 5-8). 2-Amino-5-halopyridines 1b-1d and DMFDMA with ethyl bromoacetate $\mathbf{3 a}$ also participated well in this conversion (Scheme 2, Table 2, entry 2-4). However, further expansion of the two-step onepot process to the reaction of 2-amino-5-halopyridines 1b-1d and DMF-DMA with bromoacetonitrile $\mathbf{3 b}$ or 2-bromoacetophenone $\mathbf{3 c}$ under the same reaction conditions gave the desired bicyclic products in very low yields ( $<20 \%$ ), along with some unknown by-products. Fortunately, the addition of $20 \mathrm{~mol} \% \mathrm{KI}$ into the secondstep reaction mixture significantly improved the yields because bromide group in $\mathbf{3 b}-\mathbf{3} \mathbf{c}$ could be substituted with iodide to generate more reactive iodoacetonitrile and 2-iodoacetophenone. With $\mathrm{KI}$ as an additive in the second step, we successfully conducted the reactions of $\mathbf{1 b}-\mathbf{1 d}$ and DMF-DMA with bromoacetonitrile $\mathbf{3 b}$ or 2-bromoacetophenone $3 \mathbf{c}$ to afford the corresponding 3substituted imidazo[1,2- $a$ ]pyridines $\mathbf{4 i - 4 n}$ in moderate to good yields (Scheme 2, Table 2, entries 9-14).

The results presented in Table 2 demonstrated that electron-withdrawing groups in the pyridine ring reduced the reactivity to form the products. 2-Aminopy ridine 1a afforded the desired products in high yields ranging from $74 \%$ to $89 \%$ (Tables 2 , entry 1 and 5-8). On the other hand, the reactions of $\mathbf{1 b}-\mathbf{1 d}$ (containing $\mathrm{Br}, \mathrm{Cl}$ and $\mathrm{F}$, respectively) and DMF-DMA with 3a required longer reaction time and the yields were slightly lower compared with 1a (Tables 2, entry 1-4). As for the reactions of $\mathbf{1 b}-\mathbf{1 d}$ and DMF-DMA with less reactive electrophiles $\mathbf{3 b}$ and $\mathbf{3 c}, 20 \mathrm{~mol} \% \mathrm{KI}$ was necessary for better yields (Tables 2, entry 9-14), most likely through more reactive iodide species.

\subsection{Synthesis of 3-substituted imidazo[1,2-a]pyridines $\mathbf{5 a}-\mathbf{5} \boldsymbol{f}$}

During the course of expanding the substrate scope of the two-step one-pot reaction, we found that the reaction of 2-amino-3-hydroxypyridine 1e and DMF-DMA with ethyl bromoacetate 3a under the optimal reaction conditions (without KI) gave rise to the formation of an unexpected product 5a in which the hydroxyl group underwent a substitution reaction with the active electrophile along with the imidazole ring formation. However, intermediate $\mathbf{2 e}$ was detected as the major product by TLC analysis. Notably, the substitution reaction still occurred inevitably without $\mathrm{NaHCO}_{3}$ or adding the solution of 0.8 equivalent of $\mathbf{3 a}-\mathbf{3} \mathbf{c}$ in DMF dropwise in the second step of the reaction. Finally, when 2.5 equivalent of $\mathbf{3 a}-\mathbf{3 c}$ and 2.5 equivalent of 
Table 2. Substitution of compounds 1-4 according to Scheme $2^{\mathrm{a}}$.

\begin{tabular}{|c|c|c|c|c|c|c|}
\hline Entry & 1 & $X$ & 3 & Time $(h)^{b}$ & Product & Yield $(\%)^{\mathrm{c}}$ \\
\hline 1 & 1a & $\mathrm{H}$ & $\mathbf{3 a}\left(\mathrm{R}=\mathrm{CO}_{2} \mathrm{Et}\right)$ & 1 & $4 \mathbf{a}$ & 83 \\
\hline 2 & $1 b$ & $\mathrm{Br}$ & $\mathbf{3 a}\left(\mathrm{R}=\mathrm{CO}_{2} \mathrm{Et}\right)$ & 3 & $4 b$ & 80 \\
\hline 3 & 1c & $\mathrm{Cl}$ & $\mathbf{3 a}\left(\mathrm{R}=\mathrm{CO}_{2} \mathrm{Et}\right)$ & 3 & $4 c$ & 76 \\
\hline 4 & $1 d$ & $\mathrm{~F}$ & 3a $\left(\mathrm{R}=\mathrm{CO}_{2} \mathrm{Et}\right)$ & 4 & $4 d$ & 73 \\
\hline 5 & $\mathbf{1 a}$ & $\mathrm{H}$ & $\mathbf{3 b}(\mathrm{R}=\mathrm{CN})$ & 1 & $4 e$ & 78 \\
\hline 6 & $\mathbf{1 a}$ & $\mathrm{H}$ & 3c $(\mathrm{R}=\mathrm{COPh})$ & 1 & $4 f$ & 89 \\
\hline 7 & $1 \mathbf{a}$ & $\mathrm{H}$ & 3d $\left(\mathrm{R}=4^{\prime}-\mathrm{MeO}-\mathrm{PhCO}\right)$ & 1 & $4 g$ & 74 \\
\hline 8 & $1 \mathbf{a}$ & $\mathrm{H}$ & $\mathbf{3 e}\left(\mathrm{R}=4^{\prime}-\mathrm{F}-\mathrm{PhCO}\right)$ & 1 & $4 h$ & 87 \\
\hline $9^{d}$ & $1 b$ & $\mathrm{Br}$ & $\mathbf{3 b}(\mathrm{R}=\mathrm{CN})$ & 3 & $4 \mathbf{i}$ & 75 \\
\hline $1^{\mathrm{d}}$ & 1c & $\mathrm{Cl}$ & $\mathbf{3 b}(\mathrm{R}=\mathrm{CN})$ & 3 & $4 \mathbf{j}$ & 71 \\
\hline $11^{\mathrm{d}}$ & 1d & $\mathrm{F}$ & $\mathbf{3 b}(\mathrm{R}=\mathrm{CN})$ & 4 & $4 k$ & 59 \\
\hline $12^{\mathrm{d}}$ & 1b & $\mathrm{Br}$ & $\mathbf{3 c}(\mathrm{R}=\mathrm{COPh})$ & 1.5 & 41 & 87 \\
\hline $13^{\mathrm{d}}$ & 1c & $\mathrm{Cl}$ & 3c $(\mathrm{R}=\mathrm{COPh})$ & 1.5 & $4 m$ & 78 \\
\hline $14^{\mathrm{d}}$ & 1d & $\mathrm{F}$ & 3c $(\mathrm{R}=\mathrm{COPh})$ & 2 & $4 n$ & 68 \\
\hline
\end{tabular}

${ }^{a}$ Reaction conditions: 2-Aminopyridine or its derivatives 1a-1f (1.0 mmol) and DMF-DMA (2.0 equiv) were mixed and stirred in DMF $(2 \mathrm{~mL})$ at $65^{\circ} \mathrm{C}$ for $2-3 \mathrm{~h}$. Then, active electrophiles $\mathbf{3 a}-\mathbf{3 e}$ (1.3 equiv) and $\mathrm{NaHCO}_{3}(1.5$ equiv) were added and the stirring continued at $85{ }^{\circ} \mathrm{C}$ for additional $1-4 \mathrm{~h} .{ }^{\mathrm{b}}$ Reaction time for the second step. ${ }^{\mathrm{c}}$ Isolated yield based on 1a-1f. ${ }^{\mathrm{d}} \mathrm{The}$ second step of the reaction was run with 0.2 equivalent of $\mathrm{KI}$.

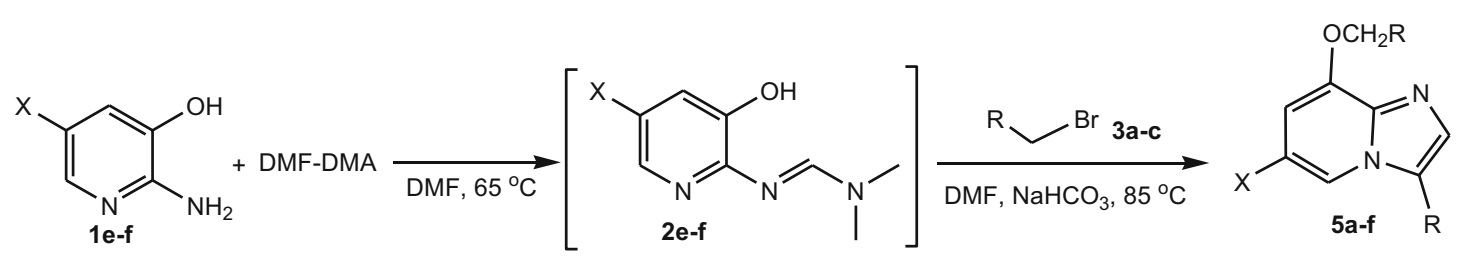

Scheme 3. Synthesis of 3-substituted imidazo[1,2-a]pyridines $\mathbf{5 a - 5 f}$.

Table 3. Substitution of compounds $\mathbf{1}, \mathbf{2}$ and $\mathbf{5}$ according to Scheme $3^{\mathrm{a}}$.

\begin{tabular}{|c|c|c|c|c|c|c|}
\hline Entry & 1 & $X$ & 3 & Time $(h)^{b}$ & Product & Yield $(\%)^{\mathrm{c}}$ \\
\hline 1 & $1 e$ & $\mathrm{H}$ & $\mathbf{3 a}\left(\mathrm{R}=\mathrm{CO}_{2} \mathrm{Et}\right)$ & 1 & $5 \mathbf{a}$ & 84 \\
\hline 2 & 1e & $\mathrm{H}$ & $\mathbf{3 b}(\mathrm{R}=\mathrm{CN})$ & 1 & $5 \mathbf{b}$ & 74 \\
\hline 3 & 1e & $\mathrm{H}$ & 3c $(\mathrm{R}=\mathrm{COPh})$ & 1 & $5 c$ & 75 \\
\hline 4 & 1f & $\mathrm{Br}$ & $\mathbf{3 a}\left(\mathrm{R}=\mathrm{CO}_{2} \mathrm{Et}\right)$ & 1 & $5 d$ & 72 \\
\hline 5 & 1f & $\mathrm{Br}$ & $\mathbf{3 b}(\mathrm{R}=\mathrm{CN})$ & 3 & $5 e$ & 64 \\
\hline 6 & 1f & $\mathrm{Br}$ & $3 \mathbf{c}(\mathrm{R}=\mathrm{COPh})$ & 3 & $\mathbf{5 f}$ & 67 \\
\hline
\end{tabular}

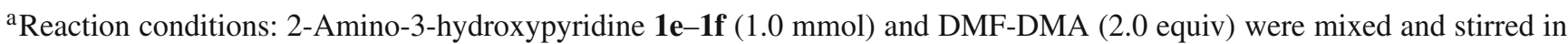
DMF $(2 \mathrm{~mL})$ at $65^{\circ} \mathrm{C}$ for $1 \mathrm{~h}$. Then, active electrophiles $\mathbf{3 b}-\mathbf{3 c}$ (2.5 equiv), $\mathrm{NaHCO}_{3}$ (3 equiv) were added and the stirring continued at $85^{\circ} \mathrm{C}$ for additional $1-3 \mathrm{~h} .{ }^{b}$ Reaction time for the second step. ${ }^{\mathrm{c}}$ Isolated yield based on 1e-1f.

$\mathrm{NaHCO}_{3}$ were used in the second step of the reaction, various 3-substituted imidazo[1,2-a]pyridines 5a-5f were obtained in good yields (Scheme 3, Table 3).

\subsection{Synthesis of 3-substituted imidazo[1,2-b]pyridazines $\mathbf{8 a}-\mathbf{8 c}$}

For further investigation, 6-chloropyridazin-3-amine 6 was also tested to synthesize the corresponding
3 -substituted fused imidazo-heterocyclic compounds. Intermediate (E)-N'-(6-chloropyridazin-3-yl)- $N, N$ dimethyformamidine $\mathbf{7}$ that formed in near quantitative yield, as detected by TLC, ${ }^{17}$ subsequently reacted with $\mathbf{3 a}-\mathbf{3 c}$ in situ and afforded the desired 3-substituted imidazo[1,2- $b$ ]pyridazines $\mathbf{8 a - 8 c}$ in moderate yields with $\mathrm{NaHCO}_{3}$ as a base and $\mathrm{KI}(20 \mathrm{~mol} \%)$ as an additive at $85{ }^{\circ} \mathrm{C}$ (Scheme 4, Table 4). This reaction represents a novel approach for the synthesis of 3 -substituted imidazo[1,2- $b]$ pyridazines. 


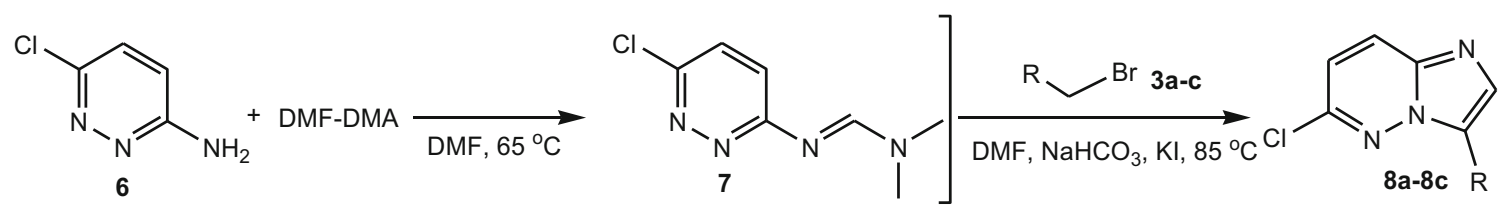

Scheme 4. Synthesis of 3-substituted imidazo[1,2-b]pyridazines 8a-8c.

Table 4. Synthesis of 3-substituted imidazo[1,2-b]pyridazines $\mathbf{8 a}-\mathbf{8} \mathbf{c}^{\mathrm{a}}$.

\begin{tabular}{llccc}
\hline Entry & \multicolumn{1}{c}{ ( } & Time $(\mathrm{h})^{\mathrm{b}}$ & Product & ${\text { Yield }(\%)^{\mathrm{c}}}^{\mathrm{c}}$ \\
\hline 1 & $\mathbf{3 a}\left(\mathrm{R}=\mathrm{CO}_{2} \mathrm{Et}\right)$ & 3 & $\mathbf{8 a}$ & 73 \\
2 & $\mathbf{3 b}(\mathrm{R}=\mathrm{CN})$ & 4 & $\mathbf{8 b}$ & 60 \\
3 & $\mathbf{3 c}(\mathrm{R}=\mathrm{COPh})$ & 3 & $\mathbf{8 c}$ & 57 \\
\hline
\end{tabular}

${ }^{a}$ Reaction conditions: 6-Chloropyridazin-3-amine 6 (1.0 mmol) and DMF-DMA (2.0 equiv) were mixed and stirred in DMF $(2 \mathrm{~mL})$ at $65^{\circ} \mathrm{C}$ for $3 \mathrm{~h}$. Then, active electrophiles $3 \mathrm{a}-3 \mathbf{c}$ ( 1.3 equiv), $\mathrm{NaHCO}_{3}$ (1.5 equiv) and $\mathrm{KI}(0.2$ equiv) were added and the stirring continued at $85^{\circ} \mathrm{C}$ for additional $3-4 \mathrm{~h} .{ }^{b}$ Reaction time for the second step. ${ }^{\mathrm{c}}$ Isolated yield based on 6 .

\section{Conclusions}

In summary, we have developed a convenient and straight-forward two-step one-pot method for the preparation of a wide variety of 3 -substituted imidazo [1,2- $a]$ pyridines and 3-substituted imidazo[1,2- $b]$ pyrid azines from readily available starting materials. The second step of the reaction is based on the condensation of intermediate $(E)$ - $N, N$-dimethy- $N^{\prime}$-(pyridin-2 -yl)-formamidine and its derivatives or $(E)-N^{\prime}$-(6-chloro pyridazin-3-yl)- $N, N$-dimethyformamidine with active electrophiles $\mathrm{RCH}_{2} \mathrm{Br}\left(\mathrm{R}=\mathrm{CN}, \mathrm{CO}_{2} \mathrm{Et}, \mathrm{COPh}, 4\right.$ '$\mathrm{MeO}-\mathrm{PhCO}$ and 4'-F-PhCO) in the presence of $\mathrm{NaHCO}_{3}$.

\section{Supplementary Information (SI)}

Copies of the original spectra $\left({ }^{1} \mathrm{H} \mathrm{NMR},{ }^{13} \mathrm{C}\right.$ NMR, mass, etc.) of all the molecules reported in the experimental section are included in Supplementary Information. Supplementary Information is available at www.ias.ac.in/chemsci.

\section{Acknowledgements}

We gratefully acknowledge the financial support by the research project (ZR2014BM014) from Shandong Provincial Natural Science Foundation and Shandong Youbang Biochemical Technology Co., LTD.

\section{References}

1. Fradley R L, Guscott M R, Bull S, Hallett D J, Goodacre S C, Wafford K A, Garrett E M, Newman R J, O'Meara G F, Whiting P J, Rosahl T W, Dawson G R, Reynolds D S and Atack J R 2007 Differential contribution of $\mathrm{GABA}_{\mathrm{A}}$ receptor subtypes to the anticonvulsant efficacy of benzodiazepine site ligands J. Psychopharmacol. 21
384; (b) Ulloora S, Shabaraya R, Aamir S and Adhikari A V 2013 New imidazo[1,2- $a$ ]pyridines carrying active pharmacophores: Synthesis and anticonvulsant studies Bioorg. Med. Chem. Lett. 231502

2. (a) Ramachandran S, Panda M, Mukherjee K, Choudhury N R, Tantry S J, Kedari C K, Ramachandran V, Sharma S, Ramya V K, Guptha S and Sambandamurthy V K 2013 Synthesis and structure activity relationship of imidazo[1,2-a]pyridine-8-carboxamides as a novel antimycobacterial lead series Bioorg. Med. Chem. Lett. 23 4996; (b) Shukla N M, Salunke D B, Yoo E, Mutz C A, Balakrishna R and David S A 2012 Antibacterial activities of Groebke-Blackburn-Bienaymé-derived imidazo[1,2-a]pyridin-3-amines Bioorg. Med. Chem. Lett. 205850

3. (a) Elkakmaoui A, Gueiffier A, Miiavet J C, Blache Y and Chapat J P 1994 Synthesis and antiviral activity of 3substituted imidazo[1,2-a]pyridine Bioorg. Med. Chem. Lett. 4 1937; (b) Lhassani M, Chavignon O, Chezal J M, Teuladeb J C, Chapat J P, Snoeckc R, Andrei G, Balzarini J, De Clercq E and Gueiffier A 1999 Synthesis and antiviral activity of imidazo[1,2-a]pyridines Eur. J. Med. Chem. 34 271; (c) Enguehard-Gueiffier C, Musiu S, Henry N, Véron J-B, Mavel S, Neyts J, Leyssen P, Paeshuyse J and Gueiffier A 2013 3Biphenylimidazo[1,2-a]pyridines or $[1,2-b]$ pyridazines and analogues, novel Flaviviridae inhibitors Eur. J. Med. Chem. 64 448; (d) Manvar P, Shaikh F, Kakadiya R, Mehariya K, Khunt R, Pandey B and Shah A 2016 Synthesis of novel imidazo[1,2-a]pyridine-4-hydroxy$2 \mathrm{H}$-coumarins by Groebke-Blackburn-Bienaymé multicomponent reaction as potential NS5B inhibitors Tetrahedron 721293

4. Marchand P, Bazin M-A, Pagniez F, Riviere G, Bodero L, Marhadour S, Nourrisson M-R, Picot C, Ruchaud S, Bach S, Baratte B, Sauvain M, Pareja D C, Vaisberg A J and Le Pape P 2015 Synthesis, antileishmanial activity and cytotoxicity of 2,3-diaryl- and 2,3,8-trisubstituted imidazo[1,2-a]pyrazines Eur. J. Med. Chem. 103 381 
5. (a) Zhou J P, Ding Y W, Zhang H B, Xu L and Dai Y 2008 Synthesis and anti-inflammatory activity of imidazo[1,2a]pyridine derivatives Chin. Chem. Lett. 19 669; (b) Lacerda R B, De Lima C K F, Da Silva L, Romeiro N C, Miranda A L P, Barreiro E J and Fraga C A M 2009 Discovery of novel analgesic and anti-inflammatory 3arylamine-imidazo[1,2-a]pyridine symbiotic prototypes Bioorg. Med. Chem. 1774

6. Moraski G C, Oliver A G, Markley L D, Cho S, Franzblau S G and Miller M J 2014 Scaffold-switching: An exploration of 5,6-fused bicyclic heteroaromatics systems to afford antituberculosis activity akin to the imidazo[1,2a]pyridine-3-carboxylate Bioorg. Med. Chem. Lett. 24 3493

7. Xi G L and Liu Z Q 2015 Introducing ferrocene into imidazo[1,2-a]pyridine by Groebke three-componentreaction for scavenging radicals and inhibiting DNA oxidation Tetrahedron 719602

8. (a) Goodacre S C, James H D, Charles H A, Philip J, Margaret K S, John M K, William M K and Michael R 2005 Imidazo-pyridine derivatives as ligands for $G A B A$ receptors U. S. Patent 0165048 A1; (b) Blackaby W P, Atack J R, Bromidge F, Castro J L, Goodacre S C, Hallett D J, Lewis R T, Marshall G R, Pike A, Smith A J, Street L J, Tattersall D F D and Waord K A 2006 Imidazo[1,2a]pyrimidines as functionally selective GABAA ligands Bioorg. Med. Chem. Lett. 16 1175; (c) Goodacre S C, Street L J, Hallett D J, Crawforth J M, Kelly S, Owens A P, Blackaby W P, Lewis R T, Stanley J, Smith A J, Ferris P, Sohal B, Cook S M, Pike A, Brown N, Wafford K A, Marshall G, Castro J L and Atack J R 2006 Imidazo[1,2- $a$ ]pyrimidines as functionally selective and orally bioavailable GABA $\alpha 2 / \alpha 3$ binding site agonists for the treatment of anxiety disorders J. Med. Chem. 49 35

9. (a) Paudler W W and Blewitt H L 1965 Ten $\pi$-electron nitrogen heterocyclic compounds.II. Bromination of imidazo[1,2-a]pyridines J. Org. Chem. 30 4081; (b) Sablayrolles C, Cros G H, Milhavet J C, Rechenq E, Chapat J P, Boucard M, Serrano J J and McNeill J H 1984 Synthesis of imidazo[1,2- $a$ ]pyrazine derivatives with uterine-relaxing, antibronchospastic, and cardiacstimulating properties J. Med. Chem. 27 206; (c) Sharma S, Saha B, Sawant D and Kundu B 2007 Synthesis of novel N-Rich polycyclic skeletons based on azoles and pyridines J. Comb. Chem. 9 783; (d) Harris A R, Nason D M, Collantes E M, Xu W J, Chi Y S, Wang Z H, Zhang B Z, Zhang Q J, Gray D L and Davoren J E 2011 Synthesis of 5-bromo-6-methyl imidazopyrazine, 5-bromo and 5chloro-6-methyl imidazopyridine using electron density surface maps to guide synthetic strategy Tetrahedron $\mathbf{6 7}$ 9063
10. (a) Podergajs S, Stanovnik B and Tisler M 1984 A new approach for the synthesis of fused imidazoles: the synthesis of 3-acyl-substituted imidazo(1,2-x)azines Synthesis 263; (b) Gome O, Salgado-Zamora H, Reyes A and Campos M E 2010 A revised approach to the synthesis of 3-acyl imidazo[1,2-a]pyridines Heterocycl. Commun. 16 99; (c) Cao H, Liu X H, Liao J Q, Huang J P, Qiu H F, Chen Q L and Chen Y Y 2014 Copper(I)and palladium(II)-catalyzed cyclizations enable a convenient synthesis of functionalized imidazo[1,2- $a$ ]pyridine aldehydes/ketones and 3-vinyl imidazo[1,2-a]pyridines J. Org. Chem. 79 11209; (d) Kim O, Jeong Y, Lee H, Hong S-S and Hong S 2011 Design and synthesis of imidazopyridine analogues as inhibitors of phosphoinositide 3-kinase signaling and angiogenesis $\mathrm{J}$. Med. Chem. 54 2455; (e) Bangade V M, Reddy B C, Thakur P B, Babu B M and Meshram H M 2013 DABCO catalyzed highly regioselective synthesis of fused imidazoheterocycles in aqueous medium Tetrahedron Lett. 54 4767

11. Kosary J, Kasztreiner E, Rabloczky G and Kurthy M 1989 Synthesis and cardiotonic activity of 2,4-diamino1.3,5-triazines Eur. J. Med. Chem. 2497

12. Fan H L, Li X, Lai X and Cao J T 2016 Method for synthesis of 6-bromoimidazo [1,2-a]pyridine-3-carboxylic acid C. N. Patent 103965190 B

13. Fan H L, Li X, Lai X S and Cao J T 2014 Process for synthesis 6-fluoroimidazo[1,2-a]pyridine-3-carboxylic acid C. N. Patent Appl. 103864786 A

14. Sucunza D, Samadi A, Chioua M, Silva D B, Yunta C, Infantes L, Carreiras M C, Soriano E and Marco-Contelles J 2011 A practical two-step synthesis of imidazo[1,2-a]pyridines from $N$-(prop-2-yn-1yl)pyridin-2-amines Chem. Commun. 475043

15. Xi N 2014 Preparation of heteroaromatic compounds as protein kinase modulators for treatment of hyperproliferative disorders U. S. Patent Appl. 0134133

16. Eastwood P R, Gonzalez R J, Gomez C E and Bach T J 2011 Fused heteroaryl imidazolone derivatives as JAK inhibitors and their preparation and use for the treatment of diseases E. P. Patent Appl. 2397482

17. (a) Cunningham I D, Blanden J S, Llor J, Muñoz L and Sharratt A P 1991 Chemistry of amidines. Part 1. Determination of the site of initial protonation in $N^{\prime}$-Pyridylformamidines J. Chem. Soc. Perkin Trans. 2 1747; (b) Huntsman E and Balsells J 2005 New Method for the General Synthesis of [1,2,4]Triazolo[1,5a]pyridines Eur. J. Org. Chem. 3761; (c) Omar G G, Héctor S Z and Elena C A 2014 Tertiary formylated amines by microwave irradiation of $N, N$-dimethyl- $N^{\prime}$ (2-pyridyl)formamidines with methyl vinyl ketone Heterocycl. Commun. 2021 